\title{
Stability Analysis of Multi-Serial-Link Mechanism Driven by Antagonistic Multiarticular Artificial Muscles
}

Yuta Ishikawa ( $\boldsymbol{\nabla}$ ishikawa.y.as@m.titech.ac.jp)

Tokyo Institute of Technology: Tokyo Kogyo Daigaku https://orcid.org/0000-0001-7294-2222

Hiroyuki Nabae

Tokyo Institute of Technology: Tokyo Kogyo Daigaku

Gen Endo

Tokyo Institute of Technology: Tokyo Kogyo Daigaku

Koichi Suzumori

Tokyo Institute of Technology: Tokyo Kogyo Daigaku

\section{Research Article}

Keywords: artificial muscle, bioinspired robot, multi-serial-link mechanism, antagonistic-muscle-driven system

Posted Date: October 25th, 2021

DOl: https://doi.org/10.21203/rs.3.rs-968693/v1

License: (c) (i) This work is licensed under a Creative Commons Attribution 4.0 International License. Read Full License 


\title{
Stability analysis of multi-serial-link mechanism driven by
} antagonistic multiarticular artificial muscles

\author{
Yuta Ishikawa $^{1 *}$, Hiroyuki Nabae ${ }^{1}$, Gen Endo $^{1}$ and Koichi Suzumori ${ }^{1}$ \\ *Corresponding author: ishikawa.y.as@m.titech.ac.jp \\ ${ }^{1}$ Department of Mechanical Engineering, Tokyo Institute of Technology, 2-12-1 Ookayama, Meguro-ku, Tokyo \\ 152-8550, Japan.
}

Abstract

Artificial multi-joint musculoskeletal systems consisting of serially connected links driven by monoarticular and multiarticular muscles, which are often inspired by vertebrates, enable robots to elicit dynamic, elegant, and flexible movements. However, serial links driven by multiarticular muscles can cause unstable motion (e.g., buckling). The stability of musculoskeletal mechanisms driven by antagonistic multi-joint muscles depends on the muscle configuration, origin/insertion of muscles, spring constants of muscles, contracting force of muscles, and other factors. We analyze the stability of a multi-serial-link mechanism driven by antagonistic multi-joint muscles aiming to prevent buckling and other undesired motions. First, we theoretically derive the potential energy of the system and the stable condition at the target point. Then, we validate the method through dynamic simulations and experiments. We confirm that we can construct a stable multiarticulate musculoskeletal system using the proposed formulation and conditions.

Keywords: artificial muscle, bioinspired robot, multi-serial-link mechanism, antagonisticmuscle-driven system

\section{Introduction}


voluntarily exert large forces and perform flexible movements with adaptability to the environment. A musculoskeletal system has two types of muscles: multiarticular muscles that span two or more joints and act on the joints simultaneously and monoarticular muscles that act on a single joint. Multiarticular muscles are thought to enable large movements involving large parts of the musculoskeletal system, while monoarticular muscles are mainly used for small movements and joint support. We have developed an artificial multiarticulate musculoskeletal robot that mimics the structure of necks of giraffes and humans $[1,2]$. We have observed that the robot curves like a bow or buckles in zigzag when multiarticular muscles, such as the longus colli, are driven without sufficient stiffness at each joint (Figs. 1 and 2). Figure 2 shows a diagram of buckling in the developed system. In an animal, monoarticular muscles, intervertebral discs, and constructions that increase joint stiffness prevent such deformation. Although musculoskeletal robots can achieve high power and flexibility, deformation remains a major challenge. Therefore, we aimed to characterize the buckling problem for a multiarticulate musculoskeletal system and use the resulting formulation in the design of musculoskeletal robots.

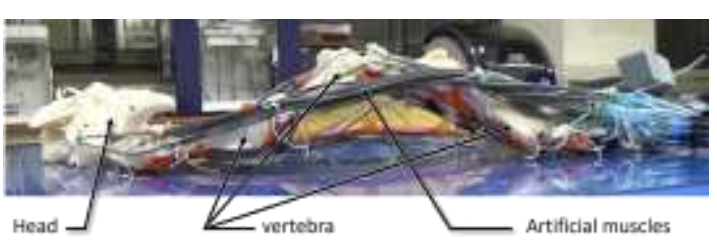



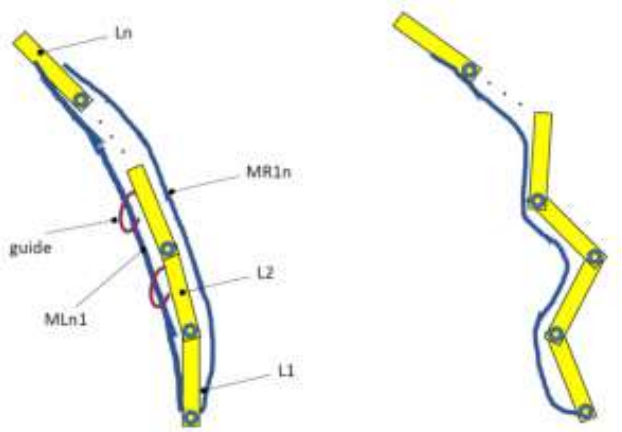

Fig. 2 Zigzag buckling of multi-serial-link mechanism driven by antagonistic multiarticular muscles without sufficient

support from monoarticular muscles

Various types of robots driven by multiarticular muscles mimicking upper and lower limbs, vertebrae,

and other anatomical structures have been proposed. The endpoint stiffness control of robots mimicking

human upper limbs has been studied for a three-bar serial-link model driven by monoarticular and

biarticular muscles $[3,4]$. In addition, a stable muscle arrangement has been studied using similar models [5]. However, as only two joints and biarticular muscles have been considered, no zigzag buckling can occur. Moreover, although the stability from the viewpoint of potential energy has been discussed [5], the tensile force of muscles has been assumed to be constant relative to length, being unsuitable to describe pneumatic artificial muscles, whose tensile force and stiffness vary according to the length and driving force, respectively.

For multiarticulate musculoskeletal lower-limb robots, control of both the reaction force from the ground and the jumping force has been conducted considering muscle elasticity [6-8]. Similar to upperlimb robots, two joints with biarticular muscles have been considered. The stiffness of the biarticular muscle and muscle model of the lower limb as a passive spring were studied in [6]. In [7], antagonistic 
muscles spanned different joints, and in [8], a model with antagonistic biarticular muscles that spanned

the same joints was derived. However, none of these studies have addressed instability in the musculoskeletal system.

Studies on multiarticulate musculoskeletal robots mimicking the upper and lower limbs have dealt with systems driven by multiarticular muscles spanning three or more joints. However, the instability conditions that we address have been neglected. Multiarticulate musculoskeletal robots consisting of three or more joints and multiarticular muscles spanning the joints have been inspired by the spine of animals such as humans $[9,10]$, lancelets [11], and snakes $[12,13]$. The robots introduced in $[9,10]$ consisted of silicone rubber to support each joint, but their instability conditions were neglected. In [11], lancelet swimming was studied using a nine-joint musculoskeletal system with seven pairs of triarticular muscles, which were arranged antagonistically. However, each antagonistic pair was driven by one motor, preventing the analysis of simultaneous contraction of antagonistic muscles and system buckling. In [12], snake locomotion was simulated, and the efficiency of multiarticular muscles was studied by changing the number of joints spanned by monoarticular up to tri-articular muscles. In [13], the superiority of multiarticular muscles over monoarticular muscles was verified with respect to force per cross-sectional area and energy efficiency. However, instability was not addressed.

In [14], the stability of multi-joint tendon-driven robots was studied in general considering the elastic energy of the tendons, and the minimum length that the tendons must be stretched was theoretically derived. However, in the corresponding wire rope-pulley system, the moment arm around each joint 
exerted by tension of each wire was assumed to be constant. Consequently, the analysis of this system

cannot be applied to musculoskeletal systems, in which the moment arm changes nonlinearly with respect to the joint angles.

Although various studies on multiarticulate musculoskeletal systems are available, few existing multiarticular muscles span three or more joints, and buckling that may occur when activating multiarticular muscles has been neglected. Moreover, the posture stability when antagonistic multiarticular muscles contract simultaneously and when the muscle stiffness changes with the driving force has not been studied. Therefore, the adequate force and stiffness of monoarticular and multiarticular muscles for stable operation of a musculoskeletal system remain to be determined.

Considering that the stiffness of most animal muscles and artificial muscles physically changes with the driving force, we aimed to clarify the required parameters of monoarticular and multiarticular muscles to avoid buckling during multiarticular muscle activation. Specifically, we analyzed the static stability of a multiarticulate musculoskeletal system using the potential energy of the system using a multi-serial-link mechanism and modeling the artificial muscles as spring elements. In addition, we considered static stability as the system convergence to a target angle and buckling instability if the system buckles in zigzag or bends uniformly like a bow. In addition to the model analysis, we verified the stability formulation through both dynamic simulations implemented in MathWorks MATLAB and experiments using McKibben artificial muscles.

\section{Methods}


Our model of musculoskeletal system is the planar multi-serial-link mechanism antagonistically driven by artificial muscles shown in Fig. 3a. Figure $3 \mathrm{~b}$ illustrates the fixed position of the multiarticular muscles spanning from joint $p$ to joint $q$. Figure 3 shows the $m$-th pair of antagonistic muscles attached to vertical fixtures at distance $a_{m}$ from the axis along each link and at distances $e_{0, m}$ and $e_{1, m}$ from each joint along each link. Thus, $e_{0, m}$ and $e_{1, m}$ represent the distance of a joint with a number and that of a joint with the subsequent number, respectively. In addition, the stiffness of the left and right sides of the $m$-th pair of antagonistic muscles, the link lengths, and the joint angles are denoted as $K_{L, m}, K_{R, m}, l_{0} \ldots l_{N}$, and $\theta_{1} \ldots \theta_{N}$, respectively. Both sides of each antagonistic muscle are driven with the same contraction force, as detailed below.

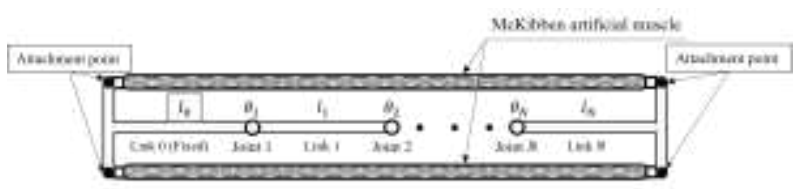

Fig. 3 Model of multi-serial-link mechanism driven by McKibben artificial muscles. a $N$-serial-link mechanism and b natural length of the $m$-th artificial muscle are denoted as $K_{L, m}, K_{R, m}$, and $L_{0, m}$, respectively 
110 We used McKibben pneumatic artificial muscles in this study. For the experiments, we used McKibben

111 muscles with a diameter of $2 \mathrm{~mm}$. This type of artificial muscle mainly consists of an inner rubber tube

112 to which air pressure is applied, and an outer sleeve of a woven fiber intersects at a certain angle. The

113 sleeve contracts through expansion in the radial direction of the inner tube.

114 Among the various formulations, the simplest equation to describe the McKibben muscle operation has been proposed by Schulte [15]:

$$
F=\frac{\pi D^{2}}{4 \sin ^{2} \theta_{0}} P\left\{3(1-\varepsilon)^{2} \cos ^{2} \theta_{0}-1\right\}
$$

117 where $F, D, P, \theta_{0}$, and $\varepsilon$ are the contraction force, diameter of the inner tube, air pressure applied to

118 the tube, woven angle of the sleeve, and contraction ratio of the artificial muscle, respectively.

119 Equation (1) is based on the virtual work principle. The work done by both the compressed air applied

to the rubber tube and the artificial muscle are assumed to be the equal. Thus, the corresponding geometric calculation neglects the elasticity of the rubber tube and sleeve strands. The maximum contraction force, which occurs when the contraction rate is 0 , seems to agree with experimental data, but the maximum contraction ratio may be inconsistent.

In the next section, we approximate the McKibben artificial muscle model as a spring, whose stiffness

Eq. (1), and the maximum contraction ratio, $\varepsilon_{\text {Max }}$, can be obtained experimentally.

$$
K=\frac{F_{\text {Max }}}{\varepsilon_{\text {Max }} S}, \quad s_{0}=S\left(1-\varepsilon_{\text {Max }}\right)
$$


pressure $P$.

We also consider the stability of the multiarticulate musculoskeletal system driven by antagonistic muscles. We focus on a straight posture, which is common for the spine. For the system to be stable, the potential energy of the entire system, $U$, must reach a local minimum at the target angles. Therefore, considering an $\mathrm{N}$-joint serial-link mechanism, a sufficient condition for stability is given by the entire potential energy, $U(\boldsymbol{\theta})\left(\boldsymbol{\theta} \in \boldsymbol{R}^{N}\right)$, satisfying the following condition:

$$
x^{T} H x>0 \quad \forall x \in R^{N}
$$

where $\boldsymbol{H} \in \boldsymbol{R}^{\boldsymbol{N} \times \boldsymbol{N}}$ is the Hessian matrix describing the second-order partial derivative of $U$.

Specifically, the element at row $i$ and column $j$ of matrix $\boldsymbol{H}$ is given by

$$
H_{i j}=\frac{\partial^{2} U}{\partial \theta_{i} \partial \theta_{j}}(1 \leq i \leq N, 1 \leq j \leq N)
$$

Equation (4) is equivalent to the following equation:

$$
\begin{gathered}
\operatorname{det}\left(\boldsymbol{A}_{k}\right)>0 \quad \forall k \in[1, N] \\
\boldsymbol{A}_{k} \equiv\left[\begin{array}{ccc}
H_{11} & \cdots & H_{1 k} \\
\vdots & \ddots & \vdots \\
H_{k 1} & \cdots & H_{k k}
\end{array}\right]
\end{gathered}
$$


produced by the antagonistic muscles:

$$
U=\sum_{m}^{M}\left\{\frac{K_{L, m}}{2}\left(\left|\boldsymbol{s}_{\boldsymbol{L}, \boldsymbol{m}}\right|-s_{0, m}\right)^{2}+\frac{K_{R, m}}{2}\left(\left|\boldsymbol{s}_{\boldsymbol{R}, \boldsymbol{m}}\right|-s_{0, m}\right)^{2}\right\}
$$

where $M$ is the number of pairs of antagonistic muscles, $\boldsymbol{s}_{\boldsymbol{L}, \boldsymbol{m}}$ and $\boldsymbol{s}_{\boldsymbol{R}, \boldsymbol{m}}$ are the endpoint vectors of the left and right sides of antagonistic muscle $m$, respectively, and $|\boldsymbol{s}|$ is the norm of vector $\boldsymbol{s}$. We set the natural length of both muscle sides to $s_{0, m}$. As shown in Fig. 3b, the endpoints of the multiarticular muscles that span from joint $p$ to joint $q$ have distances $e_{0, m}$ and $e_{1, m}$ from each joint. As the muscle endpoints are attached to the next links, $e_{0, m}$ and $e_{1, m}$ must satisfy the following constraints:

$$
0 \leq e_{0, m} \leq l_{p-1}, \quad 0 \leq e_{1, m} \leq l_{q}
$$

By using the angles of the mechanism, $\boldsymbol{s}_{\boldsymbol{L}, \boldsymbol{m}}$ and $\boldsymbol{s}_{\boldsymbol{R}, \boldsymbol{m}}$ can be described as follows:

$$
\begin{gathered}
\boldsymbol{s}_{\boldsymbol{L}, \boldsymbol{m}}=\left(\begin{array}{c}
e_{0, m}+\sum_{n=p}^{q-1} l_{n} \cos \phi_{n}+e_{1, m} \cos \phi_{q}+a_{m} \sin \phi_{q} \\
a_{m}+\sum_{n}^{q-1} l_{n} \sin \phi_{n}+e_{1, m} \sin \phi_{q}-a_{m} \cos \phi_{q}
\end{array}\right) \\
\boldsymbol{s}_{\boldsymbol{R}, \boldsymbol{m}}=\left(\begin{array}{c}
e_{0, m}+\sum_{n=p}^{q-1} l_{n} \cos \phi_{n}+e_{1, m} \cos \phi_{q}-a_{m} \sin \phi_{q} \\
-a_{m}+\sum_{n}^{q-1} l_{n} \sin \phi_{n}+e_{1, m} \cos \phi_{q}+a_{m} \cos \phi_{q}
\end{array}\right)
\end{gathered}
$$

where $\phi_{n}=\sum_{k=p}^{n} \theta_{k}$. The only difference between $\boldsymbol{s}_{\boldsymbol{L}, \boldsymbol{m}}$ and $\boldsymbol{s}_{\boldsymbol{R}, \boldsymbol{m}}$ is the change in the positive and negative signs of $a_{m}$ given the symmetry of the antagonistic muscles.

For antagonistic muscle $m$, the first- and second-order partial derivatives of its potential energy $U_{m}$ 
are respectively given by

$$
\frac{\partial U_{m}}{\partial \theta_{i}}=K_{L, m}\left(\left|\boldsymbol{s}_{L, m}\right|-s_{0, m}\right) \frac{\partial\left|\boldsymbol{s}_{L, m}\right|}{\partial \theta_{i}}+K_{R, m}\left(\left|\boldsymbol{s}_{R, m}\right|-s_{0, m}\right) \frac{\partial\left|\boldsymbol{s}_{R, m}\right|}{\partial \theta_{i}}
$$

where $U_{m}$ is assumed to be a continuous and second-order differentiable function in $\boldsymbol{R}^{N}$, and the order

of differentiation can be ignored.

As the target angles are set to $\boldsymbol{\theta}=\overrightarrow{0} \in \boldsymbol{R}^{N}$, we have

$$
\begin{aligned}
\frac{\partial^{2} U_{m}}{\partial \theta_{i} \partial \theta_{j}}( & \left.=H_{m, i j}\right)=K_{L, m} \frac{\partial\left|\boldsymbol{s}_{L, m}\right|}{\partial \theta_{i}} \frac{\partial\left|\boldsymbol{s}_{L, m}\right|}{\partial \theta_{j}}+K_{L, m}\left(\left|\boldsymbol{s}_{L, m}\right|-s_{0, m}\right) \frac{\partial^{2}\left|\boldsymbol{s}_{L, m}\right|}{\partial \theta_{i} \partial \theta_{j}} \\
& +K_{R, m} \frac{\partial\left|\boldsymbol{s}_{R, m}\right|}{\partial \theta_{i}} \frac{\partial\left|\boldsymbol{s}_{R, m}\right|}{\partial \theta_{j}}+K_{R, m}\left(\left|\boldsymbol{s}_{R, m}\right|-s_{0, m}\right) \frac{\partial^{2}\left|\boldsymbol{s}_{R, m}\right|}{\partial \theta_{i} \partial \theta_{j}}
\end{aligned}
$$

As the target angles are set to $\theta=\overrightarrow{0} \in R^{N}$, we have

66

$$
\begin{gathered}
\left|\boldsymbol{s}_{L, m}\right|=e_{0, m}+\sum_{n}^{p} l_{n}+e_{1, m} \\
\frac{\partial\left|\boldsymbol{s}_{L, m}\right|}{\partial \theta_{i}}=\frac{a_{m}\left(e_{0, m}+\sum_{n=p}^{q-1} l_{n}+e_{1, m}\right)}{e_{0, m}+\sum_{n=p}^{q-1} l_{n}+e_{1, m}}=a_{m} \\
\frac{\partial^{2}\left|\boldsymbol{s}_{L, m}\right|}{\partial \theta_{i} \partial \theta_{j}}=\frac{-\left(e_{0, m}+\sum_{n=p}^{i-1} l_{n}\right)\left(\sum_{n=j}^{q-1} l_{n}+e_{1, m}\right)}{e_{0, m}+\sum_{n}^{p} l_{n}+e_{1, m}}
\end{gathered}
$$

$$
p \leq i \leq j \leq q
$$

$$
\begin{aligned}
& \sum_{n=p}^{i-1} l_{n}=0 \text { for } i=p \\
& \sum_{n=j}^{q-1} l_{n}=0 \text { for } j=q
\end{aligned}
$$

As $U_{m}$ must satisfy Eq. (3) to be a local minimum, from Eqs. (7), (9), and (10), we obtain 


$$
K_{L, m}=K_{R, m} \quad\left(=K_{m}\right)
$$

where $\varepsilon_{\mathrm{Max}}$ is the maximum contraction ratio of the muscle, and the muscle length, $S_{m}$, can be obtained as

$$
\begin{aligned}
H_{m, i j} & =2 K_{m}\left\{a_{m}^{2}-\left(S_{m}-s_{0, m}\right) \frac{\left(e_{0, m}+\sum_{n=p}^{i-1} l_{n}\right)\left(\sum_{n=j}^{q-1} l_{n}+e_{1, m}\right)}{S_{m}}\right\} \\
& =2 K_{m}\left\{a_{m}^{2}-\left(1-\frac{s_{0, m}}{S_{m}}\right)\left(e_{0, m}+\sum_{n=p}^{i-1} l_{n}\right)\left(\sum_{n=j}^{q-1} l_{n}+e_{1, m}\right)\right\} \\
& =2 K_{m}\left\{a_{m}^{2}-\varepsilon_{\operatorname{Max}}\left(e_{0, m}+\sum_{n=p}^{i-1} l_{n}\right)\left(\sum_{n=j}^{q-1} l_{n}+e_{1, m}\right)\right\} \\
H_{i j} & =\sum_{m}^{M} 2 K_{m}\left\{a_{m}^{2}-\varepsilon_{\operatorname{Max}}\left(e_{0, m}+\sum_{n=p}^{i-1} l_{n}\right)\left(\sum_{n=j}^{q-1} l_{n}+e_{1, m}\right)\right\}
\end{aligned}
$$

$$
S_{m}=e_{0, m}+\sum_{n=p}^{q-1} l_{n}+e_{1, m}
$$

We consider contraction ratio $\varepsilon_{\text {Max }}$ to be always positive because the McKibben artificial muscles are assumed to be of contraction type. Therefore, the second term related to $\varepsilon_{\text {Max }}$ in Eq. (14) is always negative. Thus, a larger $a_{m}$ leads to more positive elements, $H_{i j}$, in the Hessian matrix, and larger $e_{0, m}, e_{1, m}$, and link lengths lead to more negative elements. From Eq. (14), we can anticipate the stability of the system by $H_{i j}$ satisfying Eq. (4) or Eq. (5).

\section{Stability of monoarticular and multiarticular muscles}



$\boldsymbol{x}=\left(0 \ldots x_{i} . .0\right)^{T}, x_{i} \in \boldsymbol{R}:$

$$
\boldsymbol{x}^{T} \boldsymbol{H} \boldsymbol{x}=H_{i i} x_{i}^{2}<0
$$

The condition above is related to the stability of joint $i$ but not to that of the entire system. Therefore, for system stability, all the diagonal elements of the Hessian matrix should be positive. span joint $p$, the corresponding Hessian matrix of potential energy is given by

$$
H_{m, i j}=\left\{\begin{array}{c}
\left.2 K_{m}\left\{a_{m}^{2}-\varepsilon_{\text {Max }} e_{0, m} e_{1, m}\right\} \text { (if } i=j=p\right) \\
0 \text { (otherwise) }
\end{array}\right.
$$

Equation (15) shows that monoarticular muscles affect only the $p$-th diagonal element of the Hessian monoarticular muscles stabilize joint $p$ when $H_{m, p p}$ is positive, which can be expressed as follows:

$$
a_{m}^{2}-\varepsilon_{\text {Max }} e_{0, m} e_{1, m}>0
$$

Equation (16) indicates that $e_{0, m}$ and/or $e_{1, m}$ must be sufficiently smaller than $a_{m}$ for the monoarticular muscles to stabilize the joint. 


$$
H_{m, i i}=\left\{\begin{array}{c}
2 K_{m}\left\{a_{m}^{2}-\varepsilon_{\operatorname{Max}}\left(e_{0, m}+\sum_{n=p}^{i-1} l_{n}\right)\left(\sum_{n=i}^{q-1} l_{n}+e_{1, m}\right)\right\} \quad(\text { if } p<i<q) \\
2 K_{m}\left\{a_{m}^{2}-\varepsilon_{\operatorname{Max}} e_{0, m}\left(\sum_{n=i}^{q-1} l_{n}+e_{1, m}\right)\right\} \quad(\text { if } i=p) \\
\left.2 K_{m}\left\{a_{m}^{2}-\varepsilon_{\operatorname{Max}}\left(e_{0, m}+\sum_{n=p}^{i-1} l_{n}\right) e_{1, m}\right\} \quad \text { (if } j=q\right) \\
0 \quad \text { (otherwise) }
\end{array}\right.
$$

208 In Eq. (17), the $p$-th and $q$-th diagonal elements become positive if $e_{0, m}$ and $e_{1, m}$ are sufficiently

209 close to 0 . In contrast, the diagonal elements for joints between $p$ and $q$ are usually negative because

$210 a_{m}$ is not much larger than the sum of the link lengths. Thus, multiarticulate antagonistic muscles cause

211 instability, especially for the joints between them.

\section{Results}

\section{Simulations}

214 We first validated our formulation through calculations and simulations implemented in MathWorks

\section{MATLAB Simulink.}

216 For the simulations and the experiments reported below, we considered a three-joint multi-serial-link

217 mechanism with four pairs of antagonistic muscles comprising three pairs of monoarticular muscles and one pair of tri-articular muscles. The parameters of the McKibben artificial muscles are listed in

Table 1 and were consistent for the simulations and experiments.

Table 1 Parameters of multi-serial-link mechanism

\begin{tabular}{c|c|c|c|c|c}
\hline \hline $\boldsymbol{l}_{\mathbf{0}}[\mathrm{mm}]$ & $l_{1}[\mathrm{~mm}]$ & $l_{2}[\mathrm{~mm}]$ & $l_{3}[\mathrm{~mm}]$ & $a[\mathrm{~mm}]$ & $h[\mathrm{~mm}]$ \\
\hline 50 & 50 & 50 & 50 & 19.5 & 9 \\
\hline
\end{tabular}


221 Therefore, we set the height gap to $h=9 \mathrm{~mm}$. Moreover, the vertical distance of all muscles from 222 links $a_{1}, \ldots, a_{4}$ was set to $19.5 \mathrm{~mm}$. Figure 4 shows a diagram of the mechanism considering the parameters listed in Table 1. Assuming that the antagonistic artificial muscles are tilted by $\psi$ in the height direction from the horizontal line, the default muscle length, $S_{m}$, and the vectors of the antagonistic muscles vary from those mentioned above. As a result, the Hessian matrix of the potential energy produced by the muscles derived from Eq. (13) can be rewritten as follows:

$$
H_{m, i j}=2 K_{m}\left\{\left(\cos ^{2} \psi+\varepsilon \sin ^{2} \psi\right) a_{m}^{2}-\varepsilon_{\operatorname{Max}}\left(e_{0, m}+\sum_{n=p}^{i-1} l_{n}\right)\left(\sum_{n=j}^{q-1} l_{n}+e_{1, m}\right)\right\}
$$

with angle $\psi$ given by

Table 2 lists the experimental parameters of the monoarticular and tri-articular muscles and of the

McKibben artificial muscles. The stiffness of the artificial muscles, $K_{m}$, was derived from Eq. (2) for

$F_{\text {Max }}$ obtained using Eq. (1). In addition, $\varepsilon_{\text {Max }}$ was determined from measurements on a tri-articular 
237 for the pressure of $0.0 \mathrm{MPa}$, which is equivalent to the muscles being not attached to the joint because

Fig. 5 .

Table 2 Parameters of McKibben artificial muscles

\begin{tabular}{c|ccc|ccc}
\hline \hline Parameter & \multicolumn{2}{|c|}{ Monoarticular muscle } & \multicolumn{3}{|c}{ Tri-articular muscle } \\
\hline$P(\mathrm{MPa})$ & 0.3 & 0.4 & 0.5 & 0.3 & 0.4 & 0.5 \\
$K_{m}(\mathrm{kN} / \mathrm{m})$ & 2.26 & 2.45 & 2.58 & 0.57 & 0.62 & 0.66 \\
$\varepsilon_{\text {Max }}(\%)$ & 13 & 16 & 19 & 13 & 16 & 19 \\
$e_{0}(\mathrm{~mm})$ & & 0 & & & 50 & \\
$e_{1}(\mathrm{~mm})$ & & 50 & & & 50 \\
\hline
\end{tabular}

Rubber tube outer diameter $D=2 \mathrm{~mm}$

Rubber tube inner diameter $d=1.16 \mathrm{~mm}$

Sleeve default angle $\theta_{0}=19^{\circ}$
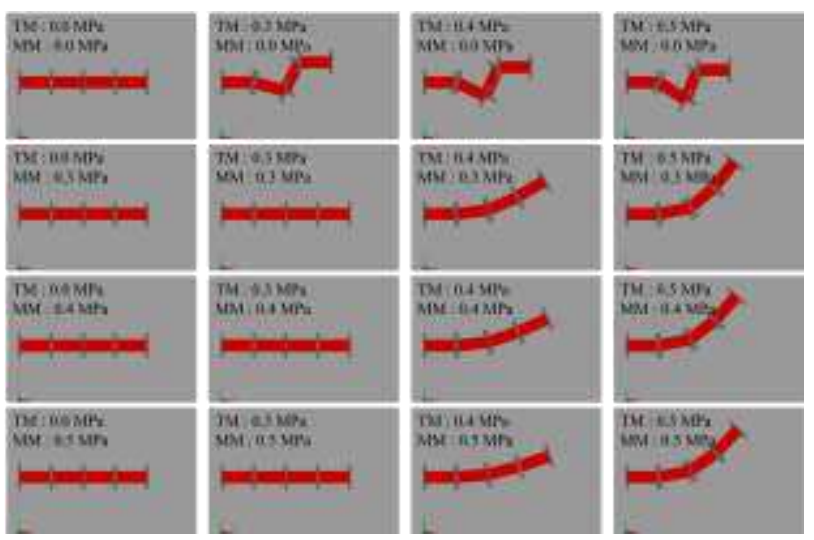

Fig. 5 Results of dynamic simulations. One spring that represents a tri-articular muscle (TM) is attached to both ends of the

Using the values in Tables 1 and 2, the determinants of $\boldsymbol{A}_{1}, \boldsymbol{A}_{2}$, and $\boldsymbol{A}_{3}$ defined in Eq. (5) are

obtained as listed in Table 3 . When no pressure $(0.0 \mathrm{MPa})$ is applied to the monoarticular muscles and 
results shown in Fig. 5. When the determinant of $\boldsymbol{A}_{1}$ is negative, instability appears as zigzag buckling.

Table 3 Determinants of $A_{1}, A_{2}$, and $A_{3}$ indicating stability in different cases

\begin{tabular}{|c|c|c|c|c|c|}
\hline & & \multicolumn{4}{|c|}{ Air pressure of tri-articular muscles (MPa) } \\
\hline & & 0.0 & 0.3 & 0.4 & 0.5 \\
\hline \multirow{12}{*}{ 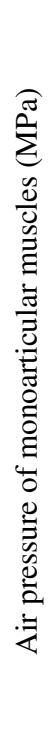 } & \multirow{3}{*}{0.0} & $\left|\boldsymbol{A}_{1}\right|=0$ & -0.68 & -1.02 & -1.37 \\
\hline & & $\left|A_{2}\right|=0$ & 0.63 & 1.28 & 2.17 \\
\hline & & $\left|A_{3}\right|=0$ & -0.35 & -1.04 & -2.32 \\
\hline & \multirow{3}{*}{0.3} & 1.68 & 0.99 & 0.65 & 0.31 \\
\hline & & 2.81 & 0.52 & -0.17 & -0.66 \\
\hline & & 4.70 & 0.42 & -0.31 & -0.51 \\
\hline & \multirow{3}{*}{0.4} & 1.82 & 1.13 & 0.80 & 0.45 \\
\hline & & 3.30 & 0.76 & -0.04 & -0.64 \\
\hline & & 5.99 & 0.76 & -0.26 & -0.67 \\
\hline & \multirow{3}{*}{0.5} & 1.91 & 1.23 & 0.89 & 0.54 \\
\hline & & 3.66 & 0.96 & 0.08 & -0.60 \\
\hline & & 7.01 & 1.07 & -0.19 & -0.77 \\
\hline
\end{tabular}

\section{Experiments}

We also verified our formulation experimentally using a three-joint multi-serial-link mechanism and

McKibben artificial muscles. A diagram and photograph of the experimental system are shown in Fig. 6.

The air pressure provided by an air compressor was adjusted using a pressure valve and applied to the monoarticular and tri-articular muscles. To reduce the effects of friction between the link and ground and in the joints, the weight of the links was reduced by fabricating them with a 3D printer, and bearings were used at each joint. The McKibben muscles used in the experiment had the parameters listed in 

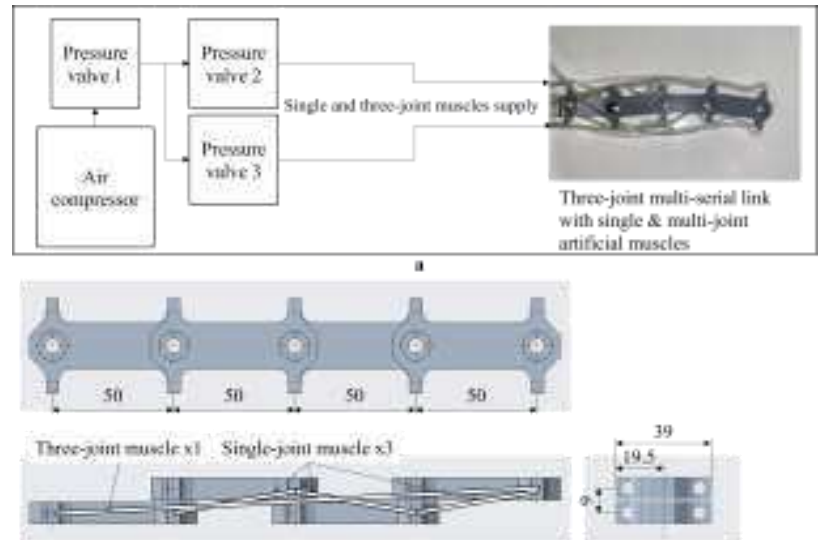

b

264 Fig. 6 a Diagram and photograph of experimental equipment and $\mathbf{b}$ diagram of multi-serial-link mechanism showing

The same cases evaluated in the simulations were tested experimentally, obtaining the experimental

results shown in Fig. 7. When the pressure applied to the monoarticular muscles is $0.0 \mathrm{MPa}$, each joint

angle changes substantially, clearly destabilizing the system at the target posture. When monoarticular

muscles are driven at any pressure except for $0.5 \mathrm{MPa}$ and tri-articular muscles are driven at 0.4 or

$0.5 \mathrm{MPa}$, the joint angles change in a smaller proportion than without using the monoarticular muscles.

When the monoarticular muscles are driven at $0.5 \mathrm{MPa}$, it is difficult to recognize the changes. When

the monoarticular muscles are driven at $0.3 \mathrm{MPa}$, no remarkable changes occur regardless of the

obtained from our formulation. 

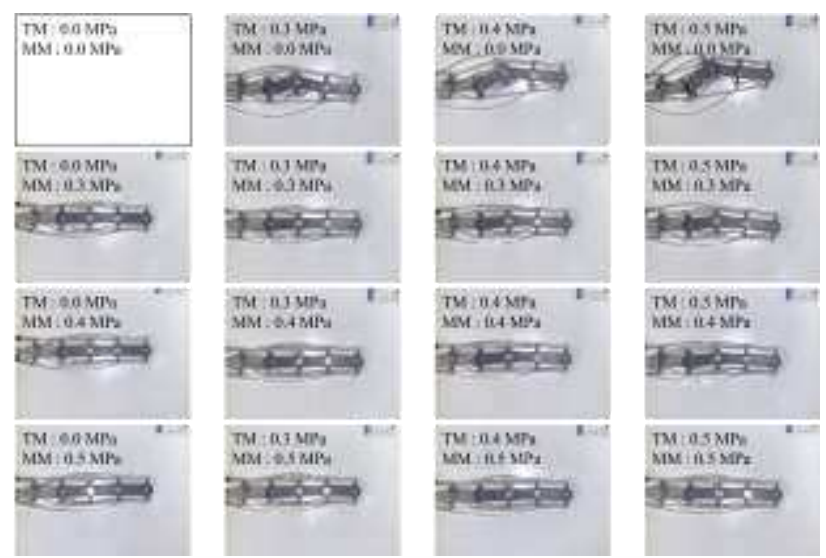

Fig. 7 Experimental results. Monoarticular and tri-articular muscles were actuated at air pressures of 0.0, 0.3, 0.4, and

$0.5 \mathrm{MPa}$

\section{Discussion}

The results in Table 3 show that the system becomes theoretically unstable at target posture $\boldsymbol{\theta}=\mathbf{0}$

for monoarticular muscles driven at $0.0 \mathrm{MPa}$ or tri-articular muscles driven at 0.4 or $0.5 \mathrm{MPa}$. In

particular, when the monoarticular muscles are inactive $(0.0 \mathrm{MPa})$, the determinant of $\boldsymbol{A}_{1}$ becomes

negative, and the serial-link mechanism buckles in zigzag in both the simulation and experimental

but the mechanism does not bend with the extent shown in the simulations. In theoretically stable

conditions, in which the tri-articular muscles are driven at 0.0 or $0.3 \mathrm{MPa}$, the mechanism does not bend and remains stable, as verified in the simulation and experimental results.

When the tri-articular muscles are driven at $0.5 \mathrm{MPa}$, the mechanism tends to stabilize as the 
monoarticular muscles are driven at higher pressures in the experiments, contradicting the simulation

291

\section{Conclusion} satisfied.

results. This inconsistent result is attributable to the effect of joint friction, which increases with the

muscle contraction forces.

In addition to the effect of joint friction, the friction caused by the interference between the artificial muscles and links tends to stabilize the posture of the mechanism. Therefore, the serial-link mechanism can remain stable at a posture around the target, at least when the theoretically stable conditions are

Considering monoarticular and multiarticular muscles in a multiarticulate musculoskeletal system, we analyze the conditions for system stability while driving the multiarticular muscles. Theoretically, we determine stability assuming that it can be reached when the potential energy produced by each articular muscle is locally minimal at the target point. In addition, the distances from the fixed point of the monoarticular muscles to the joint, $e_{0, m}$ and $e_{1, m}$, must be sufficiently small to stabilize each joint. We analyzed the stability of a three-joint multiarticulate musculoskeletal system and validated our formulation through dynamic simulations and experiments on a mechanism driven by McKibben artificial muscles. We confirmed that a sufficiently high stiffness of monoarticular muscles and a locally minimal potential energy of the system at the target posture lead to stability in the musculoskeletal mechanism consisting of serial links and multiarticulate McKibben muscles. 


\section{Declarations}

\section{Acknowledgments}

310 This work was supported by JSPS KAKENHI Grant-in-Aid for Scientific Research(A) under Grant

$311 \quad$ Number JP18H03760.

312 We would like to thank Editage (www.editage.com) for English language editing.

\section{Authors' contributions}

314 YI carried out analysis and experiment and drafted the manuscript. HN, GE, and KS were involved to

315 the study design and data interpretation. All authors revised the report, commented on the draft of the

316 manuscript, and approved the final report.

\section{$317 \quad$ Funding}

318 This work was supported by the JSPS KAKENHI Grant-in-Aid for Scientific Research(A) under Grant

$319 \quad$ Number JP18H03760.

$320 \quad$ Availability of data and materials

321 Not applicable.

\section{$322 \quad$ Competing interests}

323 The authors declare that they have no competing interest.

\section{References}

1. Niikura A, H Nabae, G Endo, M Gunji, K Mori, R Niiyama, K Suzumori (2020) Prototyping of 
musculoskeletal robot based on anatomy of a giraffe neck and static analysis of longus colli muscle. (In Japanese). (2017) Multifilament pneumatic artificial muscles to mimic the human neck. In: Proceedings of the 809-816

3. Oh S, Hori Y (2009) Development of two-degree-of-freedom control for robot manipulator with 2017 IEEE International Conference on Robotics and Biomimetics (ROBIO), Macau, Macao, pp.

4. Salvucci V, Kimura Y, Oh S, Koseki T, Hori Y (2013) Comparing approaches for actuator redundancy resolution in biarticularly-actuated robot arms. IEEE/ASME Trans Mechatron

5. Ochi H, Kino H, Tahara K, Matsutani Y (2020) Geometric conditions of a two-link-and-six muscle 00164-3

6. Ruppert F, Badri-Spröwitz A (2019) Series elastic behavior of biarticular muscle-tendon structure in a robotic leg. Front Neurorobot 13:64. doi: 10.3389/fnbot.2019.00064 
7. Kaneko T, Sekiya M, Ogata K, Sakaino S, Tsuji T (2016) Force control of a jumping musculoskeletal robot with pneumatic artificial muscles. In: 2016 IEEE/RSJ International Conference on Intelligent Robots and Systems (IROS), Daejeon, Republic of Korea, pp. 5813-5818. doi: 10.1109/IROS.2016.7759855

8. Hosoda K, Sakaguchi Y, Takayama H, Takuma T (2010) Pneumatic-driven jumping robot with anthropomorphic muscular skeleton structure. Auton Robot 28(3):307-316. doi: 10.1007/s10514009-9171-6

9. Mizuuchi I, Inaba M, Inoue H (2001) A flexible spine human-form robot-development and control of the posture of the spine. In: Proceedings of the 2001 IEEE/RSJ International Conference on Intelligent Robots and Systems, Maui, HI, pp. 2099-2104. doi: 10.1109/IROS.2001.976381

10. Mizuuchi I, Tajima R, Yoshikai T, Sato D, Nagashima K, Inaba M, Kuniyoshi Y, Inoue H (2002) The design and control of the flexible spine of a fully tendon-driven humanoid "Kenta". In: IEEE/RSJ International Conference on Intelligent Robots and Systems, Lausanne, Switzerland, pp. 2527-2532. doi: 10.1109/IRDS.2002.1041649

11. Tsuji T (2010) A model of antagonistic triarticular muscle mechanism for lancelet robot. In: 2010 11th IEEE International Workshop on Advanced Motion Control, Nagaoka, Japan, pp. 496-501. doi: 10.1109/AMC.2010.5464082

12. Kano T, Sato T, Kobayashi R, Ishiguro A (2011) Decentralized control of multi-articular snake- 
13. Astley HC (2020) The biomechanics of multi-articular muscle-tendon systems in snakes. Integr with Nonlinear Tendon Elasticity. Journal of the Robotics Society of Japan, 19(3):372-379. (In Japanese) 